\title{
Formation and Evolution of Cylindrically Diverging Detonation Waves in Gases
}

\author{
Duowen Qian ${ }^{1}$, John H.S. Lee ${ }^{1}$ and Hoi Dick $\mathrm{Ng}^{2 \dagger}$ \\ ${ }^{1}$ Department of Mechanical Engineering, McGill University, \\ Montréal, Québec, H3A 0C3, Canada \\ ${ }^{2}$ Department of Mechanical and Industrial Engineering, Concordia University, \\ Montréal, Québec, H3G 1M8, Canada \\ †Corresponding Author \\ Department of Mechanical and Industrial Engineering \\ Concordia University \\ 1455 de Maisonneuve Blvd. West \\ Montréal, H3G 1M8, Canada \\ e-mail: hoing@encs.concordia.ca \\ Tel.: (514) 848-2424 (ext. 3177) \\ Fax: (514) 848-3175
}

Winning entry submitted to

Gallery of Fluid Motion, Physics of Fluids

July, 2014 


\section{Formation and Evolution of Cylindrically Diverging Detonation Waves in Gases}

\section{Submitted by}

Duowen Qian, John H.S. Lee, McGill University, Montréal, QC, Canada Hoi Dick Ng, Concordia University, Montréal, QC, Canada

Gaseous detonations are self-sustained, combustion-driven waves traveling at supersonic velocities on the order of $2 \mathrm{~km} / \mathrm{s}$. Detonation waves are intrinsically unstable with a complex structure formed by an ensemble of interacting transverse waves. Their mutual interactions form the classical triple shock Mach interaction configuration consisted of the incident shock, Mach stem, transverse reflected wave, and the shear layer. ${ }^{1}$ The trajectories of these triple points formed along the front of a detonation wave create the characteristic fish-scale pattern or socalled detonation cells.

In this work, the cellular pattern evolution of diverging cylindrical detonations is investigated. Pre-mixed mixtures of equimolar acetylene and oxygen are used for the experiment. A detonation wave first propagates in a small tube along the direction of the $z$-axis (coming up out of the paper). It then emerges and diverges from the center of a flat channel. Images presented in Figs. 1 and 2 show a series of self-luminous, open-shutter photographs of cylindrically diverging detonations with different mixture sensitivities near the critical regime of initiation, i.e., near the critical pressure below which the diverging detonation fails. The openshutter photography technique records the time-integrated intense luminosity of the flow tracking the trajectories of the triple points and the evolution of the cellular pattern. ${ }^{2}$

For successful transmission with continuing propagation from a planar detonation to a cylindrically diverging detonation, the trajectories of the transverse waves result as two intersecting sets of logarithmic spirals. For decreasing mixture sensitivity, the cylindrically 
expanding detonation fails locally at the beginning accompanied by the disappearance of cells; however detonation is re-established subsequently by the growth of new transverse waves originating locally with a very fine structure. Artistically, the evolution of the initially failing diverging detonation and its subsequent re-initiation leads to beautiful, flowery shapes similar to blue lilies.

\section{Acknowledgement}

This research is supported by the Natural Sciences and Engineering Research Council of Canada (NSERC).

\section{References}

1'J.H.S. Lee, “The Detonation Phenomenon,” Cambridge University Press, Cambridge (2008).

${ }^{2}$ R.I. Soloukhin, “Shock Waves and Detonations in Gases," State Publishing House, Moscow (1963) 


\section{Figure Caption}

FIG. 1. An open-shutter photograph of a cylindrically diverging detonation wave near the selfpropagating limit.

FIG. 2. Images showing the propagation and failure of a cylindrical detonation wave at different initial pressures in equimolar acetylene-oxygen mixture. 


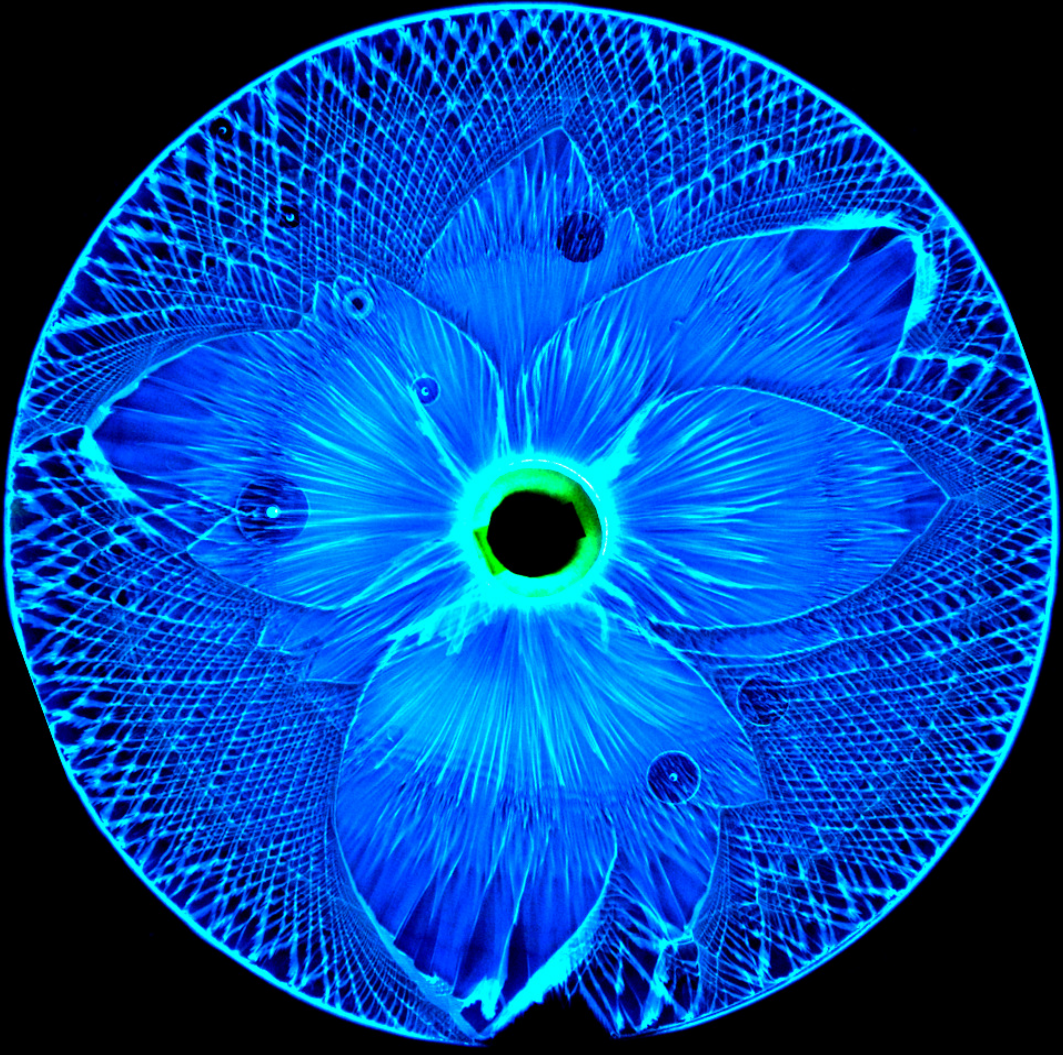




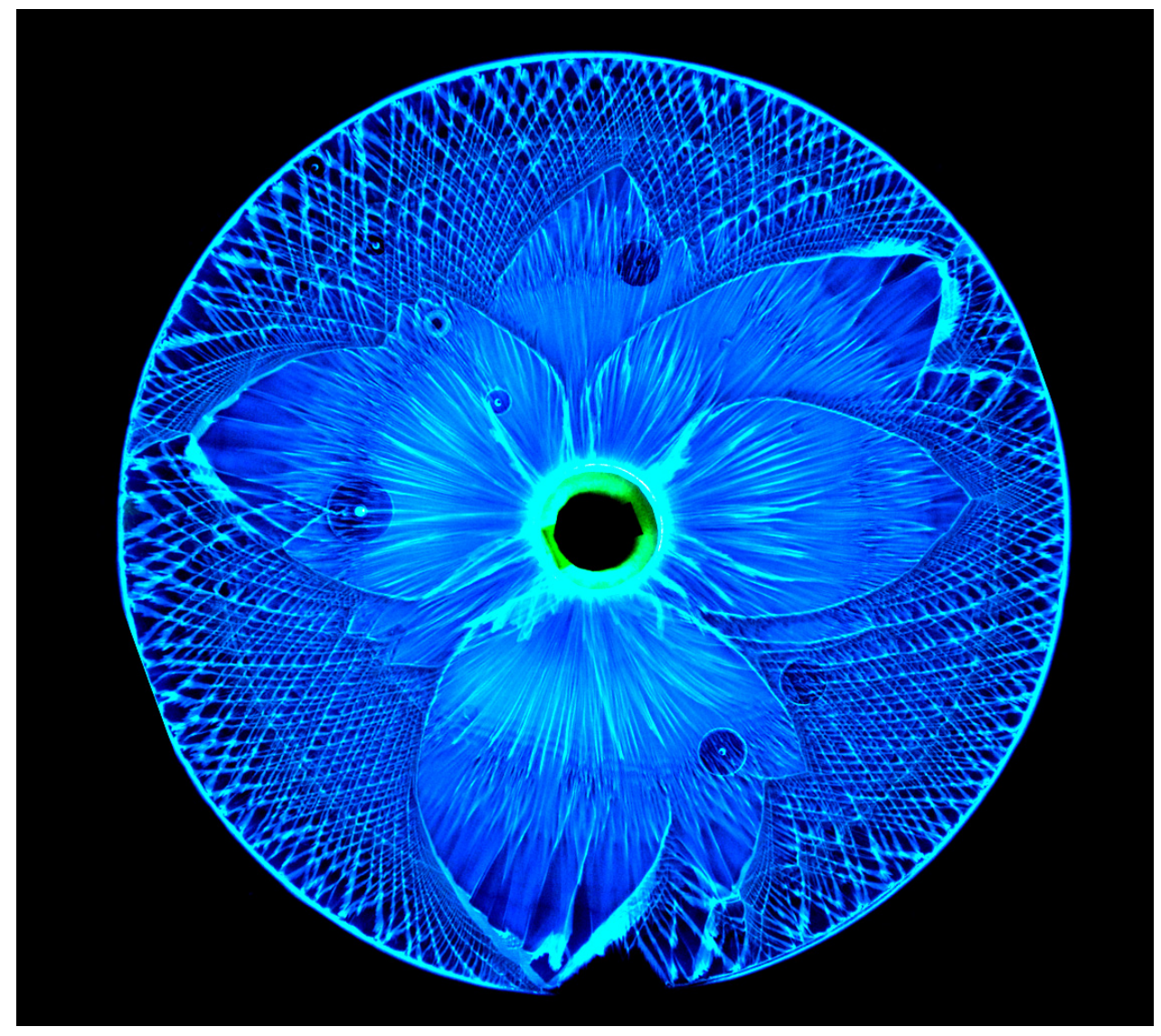




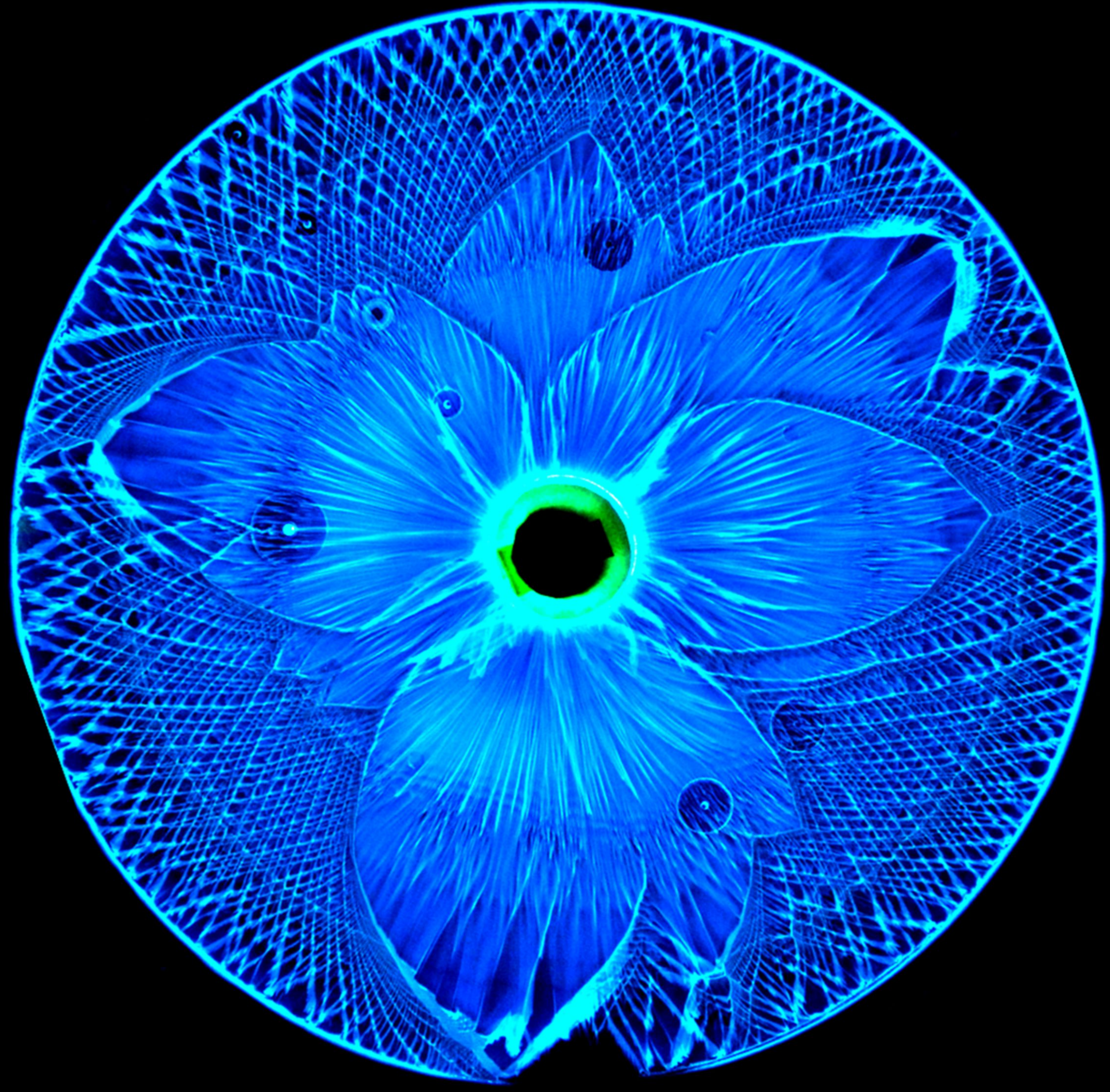




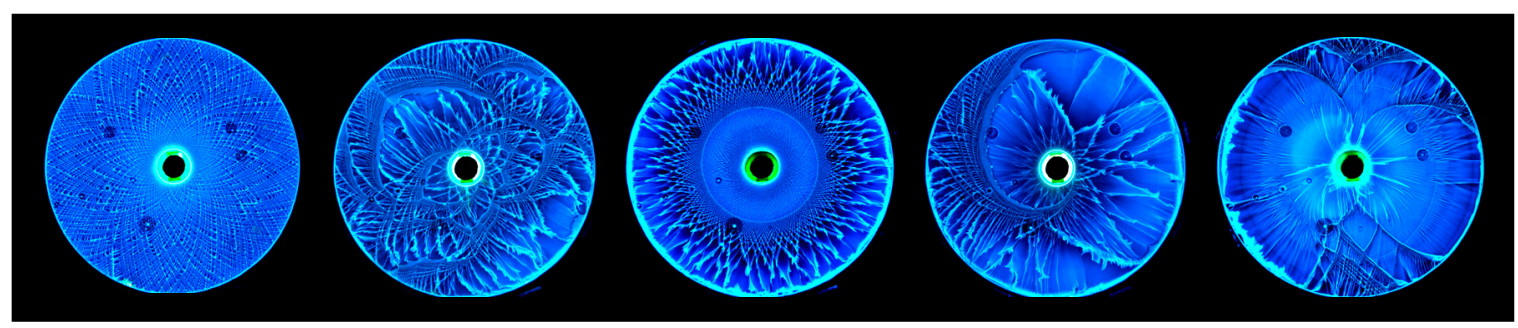


(I) KG
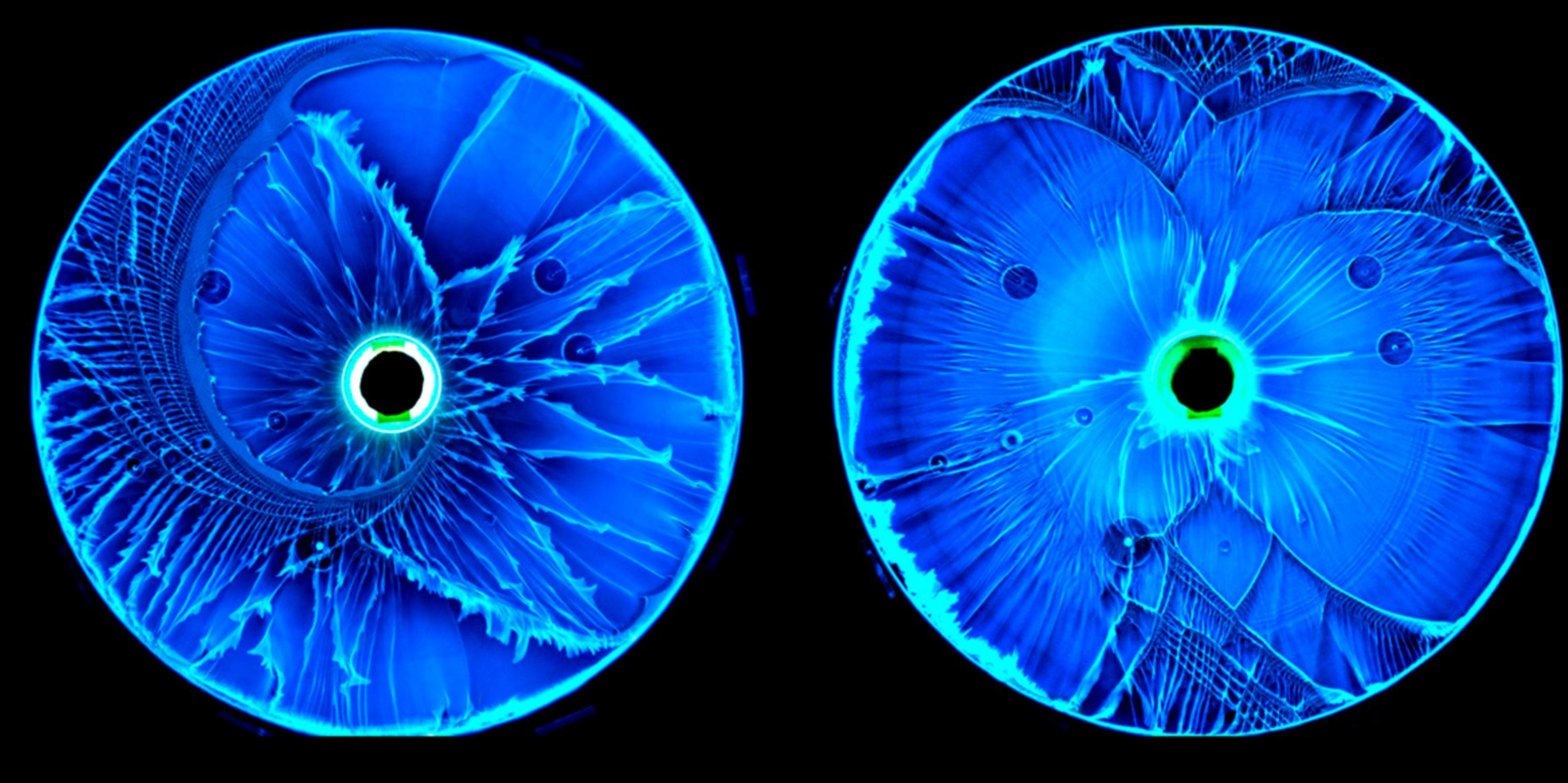